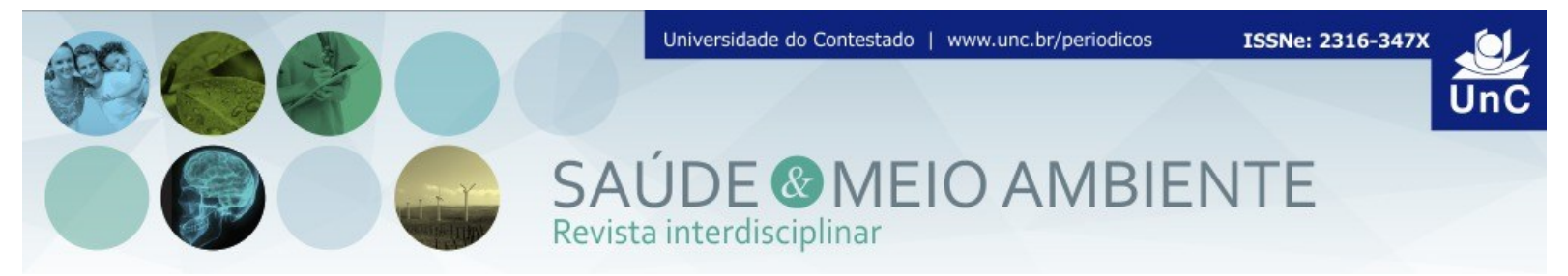

\title{
AVALIAÇÃO DO NÍVEL DE RUÍDO NAS PROXIMIDADES DO HOSPITAL ESTADUAL DE LARANJAL DO JARI (AMAPÁ)
}

\author{
Willians Lopes de Almeida ${ }^{1}$ \\ Vinícius Batista Campos ${ }^{2}$ \\ Raimundo de Moura Rolim Neto ${ }^{3}$ \\ Pablo Francisco Honorato Sampaio ${ }^{4}$ \\ Edivan Damascena Ribeiro ${ }^{5}$ \\ Jonatas Fernando Ramalho Cunha ${ }^{6}$
}

RESUMO: Este trabalho objetivou caracterizar o nível de ruído, através do nível de pressão sonora equivalente $\left(L_{\text {Aeq }}\right)$, em uma via pública, nas proximidades do Hospital Estadual do Município de Laranjal do Jari, Estado do Amapá. Para tanto, foram quantificados tais valores num período de uma semana (de 12 a 16 de maio de 2014) em dois turnos (tarde e noite). Assim, foram realizadas 30 leituras em cada período de níveis de intensidade sonora sendo 1 (um) minuto o intervalo entre cada medição, o que totalizou 60 medições por dia e 300 ao fim da semana. O cálculo do $L_{\text {Aeq }}$ se deu por meio da equação proposta pela norma brasileira 10.151/2000 e, portanto, calculou-se o nível equivalente de cada período para todos os dias. A fim de melhor interpretar os resultados e compará-los com a norma aqui exposta, fez-se a média aritmética dos valores de $L_{\text {Aeq }}$ tanto do período diurno quanto do noturno. Os resultados médios obtidos foram de 67,2 dB (A) e 66,8 dB (A), respectivamente, para tarde e noite, e mostram que o nível de pressão sonora equivalente no local não está em conformidade com os padrões dados pela norma brasileira 10.151/2000, que são de $50 \mathrm{~dB}(\mathrm{~A})$ para tarde e $45 \mathrm{~dB}(\mathrm{~A})$ para noite. Dessa forma, as pessoas que diariamente frequentam o local (na via ou hospital) ficam submetidas a um desconforto acústico, o que pode ocasionar desde estresse até cansaço físico e mental.

Palavras-chave: Acústica. Ruído. Som.

\footnotetext{
${ }^{1}$ Mestrado em Educação Agrícola pela Universidade Federal Rural do Rio de Janeiro, Brasil (2016). Professor do Instituto Federal de Educação, Ciência e Tecnologia do Amapá , Brasil. Amapá. Brasil. E-mail: willians.almeida@ifap.edu.br

${ }^{2}$ Doutorado em Engenharia Agrícola pela Universidade Federal de Campina Grande, Brasil (2012). Professor EBTT - D303 do Instituto Federal de Educação, Clência e Tecnologia do Amapá , Brasil. Amapá. Brasil. E-mail: vinicius.campos@ifap.edu.br

${ }^{3}$ Especialização em Análise e Avaliação de Impactos Ambientais pela Faculdade de Macapá, Brasil (2011). Professor do Instituto Federal do Amapá , Brasil. Mestrando em Engenharia Hidráulica e Saneamento pela Escola de Engenharia de São Carlos da Universidade de São Paulo, PPGSHS/EESC/USP. Amapá. Brasil. E-mail: raimundo.neto@ifap.edu.br

${ }^{4}$ Mestrado em Física pela Universidade Federal de Campina Grande, Brasil (2012). Professor Efetivo do Instituto Federal de Educação, Clência e Tecnologia do Amapá , Brasil Amapá. Brasil. E-mail: pablo.sampaio@ifap.edu.br

${ }^{5}$ Técnico em meio ambiente, Instituto Federal do Amapá - Campus Laranjal do Jari. Amapá. Brasil. Email: edivanli@gmail.com

${ }^{6}$ Técnico em informática. Instituto Federal do Amapá - Campus Laranjal do Jari. Amapá. Brasil. Email: jonatas.home@hotmail.com
} 


\section{NOISE LEVEL ASSESSMENT NEARBY HOSPITAL STATE OF LARANJAL OF THE JARI (AMAPA)}

ABSTRACT: This study aimed to characterize the noise level, through the equivalent sound pressure level $\left(L_{A e q}\right)$, in a public road, near the State Hospital in the city of Laranjal of the Jari, Amapa State. Therefore, these values were measured over a period of a week (on the 12th to 16th of May 2014) in two shifts (afternoon and evening). Thus, 30 readings were taken (in each period) sound intensity levels 1 (one) minute interval between each measurement, which amounted to 60 measurements per day and 300 at the end of the week. The calculation of $L_{\text {Aeq }}$ was through the equation proposed by the brazilian standard 10.151/2000 and, therefore, it was estimated the equivalent level of each period for every day. In order to better interpret the results and compare them with the standard outlined here, did the arithmetic average of the $L_{\text {Aeq }}$ values of both the daytime as the night. The average results obtained were $67.2 \mathrm{~dB}(\mathrm{~A})$ and $66.8 \mathrm{~dB}(\mathrm{~A})$, respectively, for evening and night, and show that the equivalent sound pressure level at the site does not conform to the standards given by the Brazilian standard 10.151/2000, which are $50 \mathrm{~dB}(\mathrm{~A})$ for hours and $45 \mathrm{~dB}(\mathrm{~A})$ for overnight. Thus, people who daily attend the location (on or in the hospital) shall be subject to an acoustic discomfort, which may result from stress to physical and mental fatigue.

Keywords: Acoustics. Noise. Sound.

\section{INTRODUÇÃO}

O som é uma onda mecânica que se propaga na matéria. No ar, por exemplo, essa propagação é longitudinal na faixa de frequência audível, ou seja, percebida pelo sistema auditivo humano, compreendida entre $20 \mathrm{~Hz}$ e $20 \mathrm{kHz}$ (HALLIDAY; RESNICK; WALKER, 1996). O ruído é um som que provoca desconforto mental/físico ao aparelho auditivo humano, uma vez que se origina de movimentos de vibração com diferentes frequências descrevendo um sinal aperiódico (GIANINNI et al., 2012; RUSSO, 1993 apud CARMO, 1999). De acordo com Maschke (1999) apud Sousa et. al. (2010), a medicina preventiva estabelece que um indivíduo possa estar sempre exposto a um nível equivalente de ruído não superior a $65 \mathrm{~dB}(\mathrm{~A})$.

Segundo Quadros (2004), a poluição sonora é um problema ambiental que afeta o maior número de pessoas, ficando atrás somente da poluição do ar e das águas. Diariamente as pessoas estão condicionadas a receberem sons em que os níveis de pressão sonora (NPS) encontram-se além daqueles indicados pela medicina preventiva e pelos dispositivos legais. Nesta perspectiva, a resolução CONAMA $n^{\circ} 002$ de 08 de março de 1990 veio instituir, sob a responsabilidade do IBAMA, o Programa Nacional de Educação e Controle da Poluição Sonora SILÊNCIO. Com isso, a questão do ruído excessivo passa ser encarada com mesma seriedade que a poluição dos rios ou do ar, por exemplo, visto também se tratar de saúde humana. 
Na literatura, diversos estudos (SOUSA et. al., 2010; GONÇALVES; ADISSI, 2008; NASCIMENTO et. al., 2007; ZANNIN; SZEREMETTA, 2003) mostram que os níveis de ruído que diariamente as pessoas estão submetidas, ultrapassam os valores estabelecidos pela legislação vigente, porém os estudos no município e Laranjal do Jari, e até mesmo no estado do Amapá como um todo, sobre essa temática são escassos, o que motivou ainda mais este estudo.

O município de Laranjal do Jari é relativamente novo. A lei municipal $n^{\circ} 261$ de 22 de julho de 2005 institui o código ambiental desta cidade, mas não estabelece níveis de pressão sonora máximos a que seus munícipes possam estar sujeito, mesmo a resolução CONAMA $n^{\circ}$ 002/1990 tendo em vista o bem estar e a qualidade de vida dos cidadãos. Apesar de outras normativas, como NBR 10.151/2000, já estabelecerem alguns padrões sobre a questão do ruído, a inserção deste agente poluidor em uma normativa municipal enfatizaria a preocupação do poder público para com a saúde das pessoas. Fato este que não ocorre nesta cidade.

No ambiente urbano, o ruído assume diversas fontes, de modo que a mais recorrente delas é o tráfego de veículos, visto que o número de veículos cresce cada vez mais nas cidades (MELO et. al., 2010). De acordo com Costa et. al. (2012), o município de Laranjal do Jari - AP teve, de 2002 a 2012, um aumento de $771,5 \%$ em sua frota de veículos. O que reforça ainda mais a preocupação em estudar as características do ruído e seus impactos sobre a população desta cidade.

O presente trabalho objetivou caracterizar o nível de ruído, por meio da avaliação do nível de pressão sonora equivalente $\left(L_{A e q}\right)$, em um ponto de uma via pública, nas proximidades do Hospital do município de Laranjal do Jari. A escolha deste local mostrou-se de grande relevância, pois deve-se considerar o ambiente hospitalar um lugar em que se objetive o menor incômodo possível às pessoas que ali se encontram. Inclusive os órgãos de controle e fiscalização do trânsito atribuem sinalização específica que proíbe uso de buzinas nas proximidades de tais lugares sendo, portanto, esta prática um agravante no aumento da poluição sonora nessas situações, tendo em vista que, conforme já relatado anteriormente, a cidade possui uma frota considerável de veículos os quais perfazem seus trajetos passando em frente ao hospital. Os resultados das medidas do $L_{\text {Aeq }}$ foram confrontados com os valores estabelecidos pela Norma Brasileira (NBR) 10.151/2000 sobre os níveis de ruído em áreas estritamente residencial urbana ou de hospitais ou de escolas, o qual foi caso desta pesquisa (hospital).

\section{MATERIAL E MÉTODOS}

Neste trabalho, utilizou-se como referência os valores da norma brasileira 10.151 de junho de 2000, apresentados no Quadro 1, a qual estabelece procedimentos para avaliação do ruído visando o conforto da comunidade. A avaliação do ruído consistiu na realização do cálculo do $L_{\text {Aeq }}$, o qual também é tratado na norma supracitada. 
Quadro 1 - Níveis de ruído em áreas habitadas visando o conforto da comunidade.

\begin{tabular}{lcc}
\hline \multicolumn{1}{c}{ Tipos de áreas } & \multicolumn{2}{c}{$\mathrm{L}_{\text {Aeq }}$ [dB (A)] } \\
\cline { 2 - 3 } & DIURNO & NOTURNO \\
Áreas de sítios e fazendas & 40 & 35 \\
Áreas estritamente residencial urbana ou de hospitais ou de & 50 & 45 \\
escolas & 55 & 50 \\
Área mista, predominantemente residencial & 60 & 55 \\
Área mista, com vocação comercial e administrativa & 65 & 55 \\
Área mista, com vocação recreacional & 70 & 60 \\
Área predominantemente industrial & & \\
\hline
\end{tabular}

Fonte: NBR 10.151/2000

Escolheu-se para execução deste trabalho um ponto, da Avenida Tancredo Neves (principal avenida do município de Laranjal do Jari - AP), o qual se situa nas proximidades do único hospital desta cidade. Além do mais, ao redor deste estabelecimento de saúde também se encontram pontos comerciais o que agrava a questão da poluição sonora numa área onde o ruído deve ser o mínimo possível, no entanto, o tráfego de veículos é o fator de maior relevância na emissão de ruídos no local. Os resultados foram comparados com aqueles constantes na segunda linha do Quadro 1.

Realizaram-se 30 leituras por dia, com intervalo de tempo entre cada medição de 1 (um) minuto, para cada um dos turnos (tarde e noite) durante o período compreendido de 12 a 16 de maio de 2014, o que totalizou 60 medições por dia e 300 ao fim da semana.

\section{CARACTERÍSTICAS DA AVALIAÇÃO}

Para avaliação do ruído se utilizou, como base, a norma brasileira 10.151 de junho de 2000, a qual dispõe sobre os procedimentos que devem ser adotados para a medição do nível de pressão sonora equivalente, em decibéis e ponderado em curva "A", para o conforto acústico da comunidade (ABNT, 2000).

Freitas (2006) comenta que as curvas de ponderação nascem a partir da percepção subjetiva que o ouvido humano tem, pois este não é igualmente sensível a todas as frequências. De acordo o mesmo autor, sons com frequências mais altas são percebidos de maneira aguda, enquanto que aqueles de frequências mais baixas são graves. Com isso, foram criados circuitos eletrônicos que atendessem a sensibilidade variável do ouvido humano com a frequência, e estes foram classificados como: "A", "B", "C" e "D". O circuito "A" é utilizado na medição de baixos Níveis de Pressão Sonora (NPS), os circuitos "B" e "C" para médios NPS e a curva ponderada "D" foi padronizada para medição do ruído em aeroportos.

A norma supracitada sugere que a caracterização seja feita com o uso de um sonômetro que possua a função para o cálculo do NPS equivalente, mas também 
fornece uma alternativa àqueles que não dispõem de instrumentos com esta função, ou seja, realizando-se manualmente os cálculos por meio da Equação (1).

$$
L_{A e q}=10 . \log _{10} \frac{1}{n} \sum_{i=1}^{n} 10^{\frac{L_{i}}{10}}
$$

Onde, $L_{A e q}$ é o NPS equivalente ponderado em curva "A", $L_{i}$ é uma leitura do nível de pressão sonora dada em decibéis e $\boldsymbol{n}$ representa o número total de leituras.

\section{COLETA DE DADOS}

As leituras dos níveis de pressão sonora foram feitas com auxílio de um sonômetro digital da INSTRUTHERM modelo DEC-460 (Figura 1) devidamente calibrado e configurado em tempo de resposta rápida e curva de ponderação "A".

Figura 1 - Sonômetro utilizado na coleta do nível de pressão sonora.

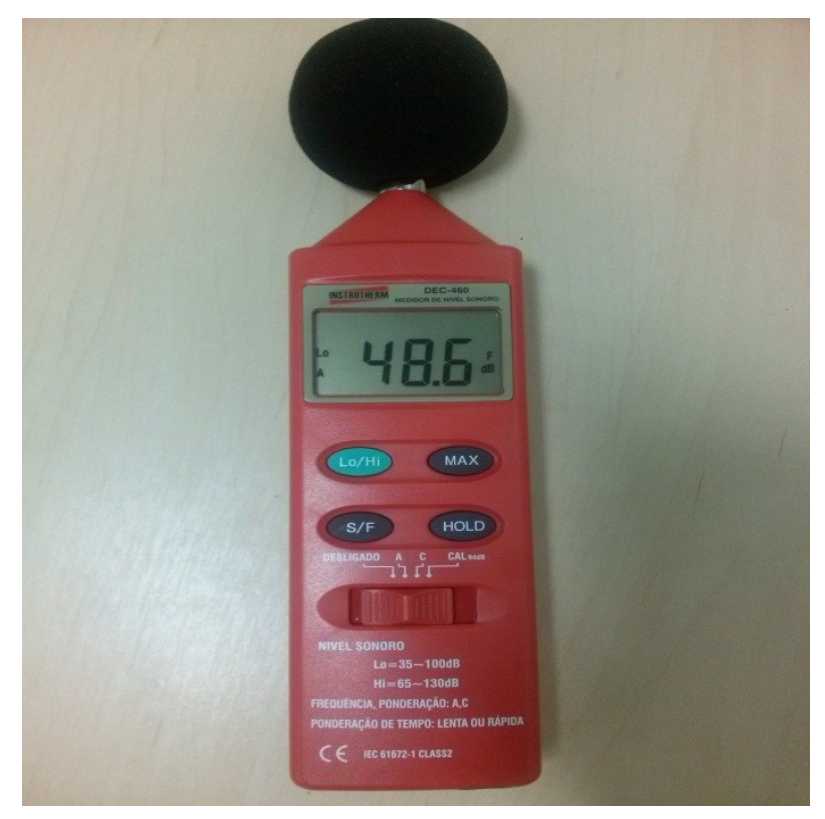

Como o sonômetro utilizado nesta pesquisa não dispunha da função de cálculo do NPS equivalente, trabalhou-se com a técnica de avaliação valendo-se da Equação (1) com uso de planilha eletrônica para efetivação do cálculo. Em todos os períodos foram realizadas 30 leituras por dia, considerando o intervalo de 1 minuto entre cada medida. Ressalta-se que nos horários em que as leituras foram feitas não houve interferências audíveis de fenômenos da natureza, tais como chuva forte, trovões e ventos fortes, conforme recomenda a NBR 10.151/2000. 


\section{RESULTADOS E DISCUSSÃO}

Antes de cada medição foram identificadas as principais fontes de ruído na localidade e constatou-se que estas estavam, basicamente, relacionadas ao tráfego de veículos (de todos os portes) e ainda na circulação de pedestres, visto que nas proximidades da área do hospital existem alguns estabelecimentos comerciais.

No Quadro 2 constam os dados dos níveis de pressão sonora observados entre $14 \mathrm{~h} 33 \mathrm{~min}$ e $15 \mathrm{~h} 03 \mathrm{~min}$ do dia 12 de maio de 2014 no turno da tarde. O nível de pressão sonora equivalente, conforme Equação (1) correspondeu a 66,2 dB (A). Já no Quadro 3 constam os dados dos níveis de pressão sonora observados entre 19h32min e 20h08min do mesmo dia, porém no turno da noite o que, levou a um nível de pressão sonora equivalente de $55,3 \mathrm{~dB}(\mathrm{~A})$.

Quadro 2 - Leituras realizadas no dia 12/05/14 (tarde) de 14h33min a 15h03min.

\begin{tabular}{|c|c|c|c|c|c|c|c|c|c|}
\hline \multicolumn{10}{|c|}{$L_{i}[\mathrm{~dB}(\mathrm{~A})]$} \\
\hline $\mathrm{L} 1$ & $\mathrm{~L} 2$ & $\mathrm{~L} 3$ & $\mathrm{~L} 4$ & $\mathrm{~L} 5$ & $\mathrm{~L} 6$ & $\mathrm{~L} 7$ & $\mathrm{~L} 8$ & $\mathrm{~L} 9$ & $\mathrm{~L} 10$ \\
\hline $\mathbf{6 3 , 3}$ & $\mathbf{6 6 , 5}$ & $\mathbf{7 3 , 9}$ & $\mathbf{5 8 , 9}$ & $\mathbf{6 0 , 2}$ & $\mathbf{6 2 , 2}$ & $\mathbf{6 3 , 0}$ & $\mathbf{6 2 , 4}$ & $\mathbf{6 3 , 7}$ & $\mathbf{6 5 , 1}$ \\
\hline $\mathrm{L} 11$ & $\mathrm{~L} 12$ & $\mathrm{~L} 13$ & $\mathrm{~L} 14$ & $\mathrm{~L} 15$ & $\mathrm{~L} 16$ & $\mathrm{~L} 17$ & $\mathrm{~L} 18$ & $\mathrm{~L} 19$ & $\mathrm{~L} 20$ \\
\hline $\mathbf{6 3 , 3}$ & $\mathbf{7 1 , 3}$ & $\mathbf{6 6 , 5}$ & $\mathbf{6 5 , 4}$ & $\mathbf{6 6 , 3}$ & $\mathbf{6 1 , 1}$ & $\mathbf{6 3 , 4}$ & $\mathbf{6 4 , 4}$ & $\mathbf{6 4 , 8}$ & $\mathbf{6 1 , 8}$ \\
\hline $\mathrm{L} 21$ & $\mathrm{~L} 22$ & $\mathrm{~L} 23$ & $\mathrm{~L} 24$ & $\mathrm{~L} 25$ & $\mathrm{~L} 26$ & $\mathrm{~L} 27$ & $\mathrm{~L} 28$ & $\mathrm{~L} 29$ & $\mathrm{~L} 30$ \\
\hline $\mathbf{7 0 , 5}$ & $\mathbf{6 4 , 8}$ & $\mathbf{6 1 , 5}$ & $\mathbf{6 2 , 8}$ & $\mathbf{6 6 , 5}$ & $\mathbf{6 2 , 5}$ & $\mathbf{6 4 , 4}$ & $\mathbf{6 8 , 8}$ & $\mathbf{6 7 , 3}$ & $\mathbf{6 5 , 6}$ \\
\hline
\end{tabular}

Quadro 3 - Leituras realizadas no dia 12/05/14 (noite) de 19h32min a 20h08min.

\begin{tabular}{|c|c|c|c|c|c|c|c|c|c|}
\hline \multicolumn{10}{|c|}{$L_{i}[\mathrm{~dB}(\mathrm{~A})]$} \\
\hline L1 & L2 & L3 & L4 & L5 & L6 & L7 & L8 & L9 & L10 \\
\hline 56,3 & 55,4 & 57,8 & 52,8 & 53,6 & 56,9 & 54,4 & 55,7 & 58,3 & 56,6 \\
\hline L11 & L12 & L13 & L14 & L15 & L16 & L17 & L18 & L19 & L20 \\
\hline 54,3 & 53,4 & 55,7 & 55,1 & 53,9 & 54,2 & 56,8 & 57,9 & 56,5 & 54,8 \\
\hline L21 & L22 & L23 & L24 & L25 & L26 & L27 & L28 & L29 & L30 \\
\hline 51,3 & 52,8 & 56,9 & 57,2 & 53,8 & 51,4 & 54,3 & 52,1 & 50,9 & 52,8 \\
\hline
\end{tabular}

No Quadro 4 constam os dados dos níveis de pressão sonora observados entre 15h16min e 15h46min do dia 13 de maio de2014 no turno da tarde, dando um nível de pressão sonora equivalente de 65,8 dB (A). No turno da noite, Quadro 5, constam os dados dos níveis de pressão sonora observados entre 19h17min e 19h52min do mesmo dia, porém com um nível de pressão sonora equivalente de $53,9 \mathrm{~dB}(\mathrm{~A})$. 
Quadro 4 - Leituras realizadas no dia 13/05/14 (tarde) de 15h16min a 15h46min.

\begin{tabular}{|c|c|c|c|c|c|c|c|c|c|}
\hline \multicolumn{10}{|c|}{$L_{i}[\mathrm{~dB}(\mathrm{~A})]$} \\
\hline $\mathrm{L} 1$ & $\mathrm{~L} 2$ & $\mathrm{~L} 3$ & $\mathrm{~L} 4$ & $\mathrm{~L} 5$ & $\mathrm{~L} 6$ & $\mathrm{~L} 7$ & $\mathrm{~L} 8$ & $\mathrm{~L} 9$ & $\mathrm{~L} 10$ \\
\hline $\mathbf{6 5 , 6}$ & $\mathbf{6 9 , 7}$ & $\mathbf{6 5 , 6}$ & $\mathbf{6 0 , 6}$ & $\mathbf{6 3 , 5}$ & $\mathbf{6 3 , 9}$ & $\mathbf{6 3 , 1}$ & $\mathbf{6 1 , 4}$ & $\mathbf{6 5 , 6}$ & $\mathbf{6 6 , 5}$ \\
\hline $\mathrm{L} 11$ & $\mathrm{~L} 12$ & $\mathrm{~L} 13$ & $\mathrm{~L} 14$ & $\mathrm{~L} 15$ & $\mathrm{~L} 16$ & $\mathrm{~L} 17$ & $\mathrm{~L} 18$ & $\mathrm{~L} 19$ & $\mathrm{~L} 20$ \\
\hline $\mathbf{6 7 , 1}$ & $\mathbf{6 5 , 2}$ & $\mathbf{6 1 , 7}$ & $\mathbf{6 5 , 1}$ & $\mathbf{7 0 , 9}$ & $\mathbf{6 4 , 4}$ & $\mathbf{6 6 , 2}$ & $\mathbf{6 6 , 9}$ & $\mathbf{6 3 , 7}$ & $\mathbf{6 3 , 3}$ \\
\hline $\mathrm{L} 21$ & $\mathrm{~L} 22$ & $\mathrm{~L} 23$ & $\mathrm{~L} 24$ & $\mathrm{~L} 25$ & $\mathrm{~L} 26$ & $\mathrm{~L} 27$ & $\mathrm{~L} 28$ & $\mathrm{~L} 29$ & $\mathrm{~L} 30$ \\
\hline $\mathbf{6 2 , 2}$ & $\mathbf{6 7 , 5}$ & $\mathbf{6 1 , 0}$ & $\mathbf{5 9 , 7}$ & $\mathbf{6 6 , 3}$ & $\mathbf{6 1 , 0}$ & $\mathbf{6 3 , 6}$ & $\mathbf{5 9 , 0}$ & $\mathbf{7 2 , 8}$ & $\mathbf{6 1 , 8}$ \\
\hline
\end{tabular}

Quadro 5 - Leituras realizadas no dia 13/05/14 (noite) de 19h17min a 19h52min.

\begin{tabular}{|c|c|c|c|c|c|c|c|c|c|}
\hline \multicolumn{10}{|c|}{$L_{i}[\mathrm{~dB}(\mathrm{~A})]$} \\
\hline $\mathrm{L} 1$ & $\mathrm{~L} 2$ & $\mathrm{~L} 3$ & $\mathrm{~L} 4$ & $\mathrm{~L} 5$ & $\mathrm{~L} 6$ & $\mathrm{~L} 7$ & $\mathrm{~L} 8$ & $\mathrm{~L} 9$ & $\mathrm{~L} 10$ \\
\hline $\mathbf{5 0 , 3}$ & $\mathbf{5 2 , 4}$ & $\mathbf{5 3 , 8}$ & $\mathbf{4 9 , 7}$ & $\mathbf{5 1 , 5}$ & $\mathbf{5 3 , 6}$ & $\mathbf{5 5 , 8}$ & $\mathbf{5 6 , 4}$ & $\mathbf{5 5 , 2}$ & $\mathbf{5 5 , 8}$ \\
\hline $\mathrm{L} 11$ & L12 & L13 & L14 & L15 & L16 & L17 & L18 & L19 & L20 \\
\hline $\mathbf{5 4 , 7}$ & $\mathbf{5 6 , 9}$ & $\mathbf{5 5 , 4}$ & $\mathbf{5 3 , 8}$ & $\mathbf{5 4 , 7}$ & $\mathbf{5 5 , 6}$ & $\mathbf{5 7 , 8}$ & $\mathbf{5 6 , 5}$ & $\mathbf{5 4 , 3}$ & $\mathbf{5 2 , 1}$ \\
\hline $\mathrm{L} 21$ & L22 & L23 & L24 & L25 & L26 & L27 & L28 & L29 & L30 \\
\hline $\mathbf{5 0 , 3}$ & $\mathbf{5 2 , 7}$ & $\mathbf{4 9 , 8}$ & $\mathbf{4 9 , 6}$ & $\mathbf{4 9 , 3}$ & $\mathbf{5 0 , 2}$ & $\mathbf{5 2 , 3}$ & $\mathbf{5 0 , 5}$ & $\mathbf{5 2 , 7}$ & $\mathbf{5 1 , 8}$ \\
\hline
\end{tabular}

No Quadro 6 constam os dados dos níveis de pressão sonora observados entre $15 \mathrm{~h} 42 \mathrm{~min}$ e $16 \mathrm{~h} 12 \mathrm{~min}$ do dia 14 de maio2014 no turno da tarde, dando um nível de pressão sonora equivalente de 66,0 dB (A). No turno da noite, Quadro 7, registrou-se níveis de pressão sonora observados entre 19h10min e 19h48min do mesmo dia, o que levou a um nível de pressão sonora equivalente de 56,7 dB (A).

Quadro 6 - Leituras realizadas no dia 14/05/14 (tarde) de 15h42min a 16h42min.

\begin{tabular}{|c|c|c|c|c|c|c|c|c|c|}
\hline \multicolumn{10}{|c|}{$L_{i}[\mathrm{~dB}(\mathrm{~A})]$} \\
\hline $\mathrm{L} 1$ & $\mathrm{~L} 2$ & $\mathrm{~L} 3$ & $\mathrm{~L} 4$ & $\mathrm{~L} 5$ & $\mathrm{~L} 6$ & $\mathrm{~L} 7$ & $\mathrm{~L} 8$ & $\mathrm{~L} 9$ & $\mathrm{~L} 10$ \\
\hline $\mathbf{5 6 , 8}$ & $\mathbf{6 3 , 9}$ & $\mathbf{6 6 , 4}$ & $\mathbf{6 7 , 1}$ & $\mathbf{6 8 , 9}$ & $\mathbf{6 6 , 0}$ & $\mathbf{6 4 , 4}$ & $\mathbf{6 2 , 9}$ & $\mathbf{6 4 , 9}$ & $\mathbf{6 2 , 4}$ \\
\hline $\mathrm{L} 11$ & $\mathrm{~L} 12$ & $\mathrm{~L} 13$ & $\mathrm{~L} 14$ & $\mathrm{~L} 15$ & $\mathrm{~L} 16$ & $\mathrm{~L} 17$ & $\mathrm{~L} 18$ & $\mathrm{~L} 19$ & $\mathrm{~L} 20$ \\
\hline $\mathbf{6 2 , 8}$ & $\mathbf{6 5 , 1}$ & $\mathbf{6 9 , 8}$ & $\mathbf{6 3 , 3}$ & $\mathbf{7 1 , 3}$ & $\mathbf{6 6 , 5}$ & $\mathbf{6 8 , 5}$ & $\mathbf{6 5 , 8}$ & $\mathbf{6 7 , 0}$ & $\mathbf{6 4 , 9}$ \\
\hline $\mathrm{L} 21$ & $\mathrm{~L} 22$ & $\mathrm{~L} 23$ & $\mathrm{~L} 24$ & $\mathrm{~L} 25$ & $\mathrm{~L} 26$ & $\mathrm{~L} 27$ & $\mathrm{~L} 28$ & $\mathrm{~L} 29$ & $\mathrm{~L} 30$ \\
\hline $\mathbf{6 4 , 4}$ & $\mathbf{6 3 , 2}$ & $\mathbf{6 6 , 2}$ & $\mathbf{6 1 , 8}$ & $\mathbf{6 5 , 4}$ & $\mathbf{6 8 , 3}$ & $\mathbf{6 6 , 2}$ & $\mathbf{6 4 , 2}$ & $\mathbf{6 3 , 4}$ & $\mathbf{6 4 , 6}$ \\
\hline
\end{tabular}


Quadro 7 - Leituras realizadas no dia 14/05/14 (noite) de 19h10min a 19h48min.

\begin{tabular}{|c|c|c|c|c|c|c|c|c|c|}
\hline \multicolumn{10}{|c|}{$L_{\boldsymbol{i}}[\mathrm{dB}(\mathbf{A})]$} \\
\hline L1 & L2 & L3 & L4 & L5 & L6 & L7 & L8 & L9 & L10 \\
\hline $\mathbf{5 5 , 8}$ & $\mathbf{5 6 , 3}$ & $\mathbf{5 7 , 9}$ & $\mathbf{5 6 , 4}$ & $\mathbf{5 5 , 1}$ & $\mathbf{5 4 , 8}$ & $\mathbf{5 3 , 7}$ & $\mathbf{5 0 , 2}$ & $\mathbf{5 1 , 6}$ & $\mathbf{5 2 , 5}$ \\
\hline L11 & L12 & L13 & L14 & L15 & L16 & L 17 & L 18 & L 19 & L20 \\
\hline $\mathbf{5 4 , 9}$ & $\mathbf{5 4 , 6}$ & $\mathbf{5 7 , 8}$ & $\mathbf{5 6 , 3}$ & $\mathbf{5 7 , 7}$ & $\mathbf{5 9 , 4}$ & $\mathbf{5 8 , 6}$ & $\mathbf{5 7 , 8}$ & $\mathbf{5 8 , 1}$ & $\mathbf{5 8 , 5}$ \\
\hline L21 & L22 & L23 & L24 & L25 & L26 & L27 & L28 & L29 & L30 \\
\hline $\mathbf{5 6 , 3}$ & $\mathbf{5 6 , 7}$ & $\mathbf{5 4 , 8}$ & $\mathbf{5 6 , 5}$ & $\mathbf{5 9 , 6}$ & $\mathbf{5 8 , 1}$ & $\mathbf{5 8 , 8}$ & $\mathbf{5 6 , 6}$ & $\mathbf{5 4 , 9}$ & $\mathbf{5 5 , 3}$ \\
\hline
\end{tabular}

No Quadro 8 constam os dados dos níveis de pressão sonora observados entre $16 \mathrm{~h} 20 \mathrm{~min}$ e $16 \mathrm{~h} 51 \mathrm{~min}$ do dia 15 de maio2014 no turno da tarde e o nível de pressão sonora equivalente correspondeu a 68,2 dB (A). Já para o Quadro 9 constam os dados dos níveis de pressão sonora observados entre 19h22mim e 19h58mim do mesmo dia, porém no turno da noite o que, levou a um nível de pressão sonora equivalente de $58,3 \mathrm{~dB}(\mathrm{~A})$.

Quadro 8 - Leituras realizadas no dia 15/05/14 (tarde) de 16h20min a 16h51min.

\begin{tabular}{|c|c|c|c|c|c|c|c|c|c|}
\hline \multicolumn{10}{|c|}{$L_{\boldsymbol{i}}[\mathrm{dB}(\mathrm{A})]$} \\
\hline $\mathrm{L} 1$ & $\mathrm{~L} 2$ & $\mathrm{~L} 3$ & $\mathrm{~L} 4$ & $\mathrm{~L} 5$ & $\mathrm{~L} 6$ & $\mathrm{~L} 7$ & $\mathrm{~L} 8$ & $\mathrm{~L} 9$ & $\mathrm{~L} 10$ \\
\hline $\mathbf{6 9 , 2}$ & $\mathbf{6 6 , 1}$ & $\mathbf{6 8 , 5}$ & $\mathbf{6 6 , 8}$ & $\mathbf{6 6 , 7}$ & $\mathbf{6 7 , 9}$ & $\mathbf{7 3 , 8}$ & $\mathbf{6 5 , 9}$ & $\mathbf{6 6 , 6}$ & $\mathbf{6 5 , 8}$ \\
\hline $\mathrm{L} 11$ & $\mathrm{~L} 12$ & $\mathrm{~L} 13$ & $\mathrm{~L} 14$ & $\mathrm{~L} 15$ & $\mathrm{~L} 16$ & $\mathrm{~L} 17$ & $\mathrm{~L} 18$ & $\mathrm{~L} 19$ & $\mathrm{~L} 20$ \\
\hline $\mathbf{6 6 , 6}$ & $\mathbf{6 6 , 9}$ & $\mathbf{6 8 , 7}$ & $\mathbf{6 2 , 2}$ & $\mathbf{6 9 , 0}$ & $\mathbf{6 4 , 7}$ & $\mathbf{7 0 , 4}$ & $\mathbf{6 4 , 2}$ & $\mathbf{6 7 , 2}$ & $\mathbf{6 7 , 4}$ \\
\hline $\mathrm{L} 21$ & $\mathrm{~L} 22$ & $\mathrm{~L} 23$ & $\mathrm{~L} 24$ & $\mathrm{~L} 25$ & $\mathrm{~L} 26$ & $\mathrm{~L} 27$ & $\mathrm{~L} 28$ & $\mathrm{~L} 29$ & $\mathrm{~L} 30$ \\
\hline $\mathbf{6 0 , 4}$ & $\mathbf{6 6 , 7}$ & $\mathbf{6 4 , 6}$ & $\mathbf{6 8 , 1}$ & $\mathbf{6 2 , 1}$ & $\mathbf{7 4 , 3}$ & $\mathbf{6 4 , 9}$ & $\mathbf{6 7 , 4}$ & $\mathbf{6 8 , 6}$ & $\mathbf{7 1 , 2}$ \\
\hline
\end{tabular}

Quadro 1 - Leituras realizadas no dia 15/05/14 (noite) de 19h22mim a 19h58mim

\begin{tabular}{|c|c|c|c|c|c|c|c|c|c|}
\hline \multicolumn{10}{|c|}{$L i[\mathrm{~dB}(\mathrm{~A})]$} \\
\hline L1 & $\mathrm{L} 2$ & L3 & L4 & L5 & L6 & L7 & L8 & L9 & L10 \\
\hline 59,8 & 57,6 & 57,4 & 58,6 & 59,3 & 59,8 & 61,2 & 60,5 & 59,5 & 58,6 \\
\hline L11 & L12 & L13 & L14 & L15 & L16 & L17 & L18 & L19 & L20 \\
\hline 56,7 & 55,4 & 54,8 & 58,4 & 59,7 & 58,4 & 58,9 & 57,6 & 57,1 & 59,3 \\
\hline $\mathrm{L} 21$ & L22 & L23 & L24 & $\mathrm{L} 25$ & L26 & L27 & L28 & L29 & L30 \\
\hline 58,3 & 57,6 & 54,8 & 55,9 & 58,7 & 57,4 & 56,8 & 56,6 & 57,1 & 59,4 \\
\hline
\end{tabular}

No Quadro 10 constam os níveis de pressão sonora observados entre $16 \mathrm{~h} 04 \mathrm{~min}$ e $16 \mathrm{~h} 35 \mathrm{~min}$ do dia 16 de maio de2014 no turno da tarde, dando um nível de pressão sonora equivalente de 70,0 dB (A). No turno da noite (Quadro 11), foram para registrados níveis de pressão sonora observados entre $19 \mathrm{~h} 28 \mathrm{~min}$ e $20 \mathrm{~h} 04 \mathrm{~min}$ do mesmo dia, porém com um nível de pressão sonora equivalente de 59,8 dB (A). 
Quadro 10 - Leituras realizadas no dia 16/05/14 (tarde) de 16h04min a 16h35min.

\begin{tabular}{|c|c|c|c|c|c|c|c|c|c|}
\hline \multicolumn{10}{|c|}{$L_{i}[\mathrm{~dB}(\mathrm{~A})]$} \\
\hline $\mathrm{L} 1$ & $\mathrm{~L} 2$ & $\mathrm{~L} 3$ & $\mathrm{~L} 4$ & $\mathrm{~L} 5$ & $\mathrm{~L} 6$ & $\mathrm{~L} 7$ & $\mathrm{~L} 8$ & $\mathrm{~L} 9$ & $\mathrm{~L} 10$ \\
\hline $\mathbf{6 9 , 9}$ & $\mathbf{7 4 , 9}$ & $\mathbf{7 0 , 4}$ & $\mathbf{6 3 , 1}$ & $\mathbf{6 5 , 6}$ & $\mathbf{6 6 , 8}$ & $\mathbf{6 6 , 0}$ & $\mathbf{7 5 , 9}$ & $\mathbf{6 6 , 6}$ & $\mathbf{6 2 , 4}$ \\
\hline $\mathrm{L} 11$ & $\mathrm{~L} 12$ & $\mathrm{~L} 13$ & $\mathrm{~L} 14$ & $\mathrm{~L} 15$ & $\mathrm{~L} 16$ & $\mathrm{~L} 17$ & $\mathrm{~L} 18$ & $\mathrm{~L} 19$ & $\mathrm{~L} 20$ \\
\hline $\mathbf{6 8 , 7}$ & $\mathbf{6 0 , 1}$ & $\mathbf{6 9 , 5}$ & $\mathbf{6 8 , 4}$ & $\mathbf{6 5 , 5}$ & $\mathbf{6 8 , 4}$ & $\mathbf{6 7 , 8}$ & $\mathbf{7 1 , 9}$ & $\mathbf{6 7 , 9}$ & $\mathbf{6 3 , 7}$ \\
\hline $\mathrm{L} 21$ & $\mathrm{~L} 22$ & $\mathrm{~L} 23$ & $\mathrm{~L} 24$ & $\mathrm{~L} 25$ & $\mathrm{~L} 26$ & $\mathrm{~L} 27$ & $\mathrm{~L} 28$ & $\mathrm{~L} 29$ & $\mathrm{~L} 30$ \\
\hline $\mathbf{6 8 , 2}$ & $\mathbf{6 9 , 7}$ & $\mathbf{6 9 , 1}$ & $\mathbf{6 6 , 7}$ & $\mathbf{7 7 , 7}$ & $\mathbf{6 3 , 9}$ & $\mathbf{7 2 , 2}$ & $\mathbf{6 8 , 6}$ & $\mathbf{6 7 , 3}$ & $\mathbf{6 5 , 6}$ \\
\hline
\end{tabular}

Quadro 11 - Leituras realizadas no dia 16/05/14 (noite) de 19h28min a 20h04min.

\begin{tabular}{|c|c|c|c|c|c|c|c|c|c|}
\hline \multicolumn{10}{|c|}{$L_{i}[\mathrm{~dB}(\mathrm{~A})]$} \\
\hline L1 & L2 & L3 & L4 & L5 & L6 & L7 & L8 & L9 & L10 \\
\hline $\mathbf{5 7 , 6}$ & $\mathbf{5 8 , 6}$ & $\mathbf{5 8 , 3}$ & $\mathbf{5 9 , 7}$ & $\mathbf{5 9 , 4}$ & $\mathbf{6 0 , 2}$ & $\mathbf{6 1 , 5}$ & $\mathbf{6 2 , 4}$ & $\mathbf{6 0 , 5}$ & $\mathbf{6 0 , 7}$ \\
\hline L11 & L12 & L13 & L14 & L15 & L16 & L17 & L18 & L19 & L20 \\
\hline $\mathbf{5 9 , 7}$ & $\mathbf{5 8 , 6}$ & $\mathbf{5 9 , 4}$ & $\mathbf{5 9 , 5}$ & $\mathbf{5 9 , 7}$ & $\mathbf{5 8 , 4}$ & $\mathbf{5 7 , 6}$ & $\mathbf{5 8 , 9}$ & $\mathbf{5 9 , 3}$ & $\mathbf{5 9 , 7}$ \\
\hline L21 & L22 & L23 & L24 & L25 & L26 & L27 & L28 & L29 & L30 \\
\hline $\mathbf{5 9 , 6}$ & $\mathbf{5 9 , 1}$ & $\mathbf{6 2 , 3}$ & $\mathbf{6 1 , 8}$ & $\mathbf{5 9 , 6}$ & $\mathbf{5 8 , 7}$ & $\mathbf{5 8 , 1}$ & $\mathbf{5 9 , 2}$ & $\mathbf{5 9 , 9}$ & $\mathbf{6 0 , 4}$ \\
\hline
\end{tabular}

De posse dos níveis de pressão sonora equivalente de cada dia e a fim de melhor interpretar os dados com base nos valores do Quadro 1, procedeu-se com o cálculo da média aritmética desses, conforme demonstrado nas Figuras $2 \mathrm{a}$ e $2 \mathrm{~b}$, respectivamente, para os turnos da tarde e noite.

Figura 2 - Média dos níveis de pressão sonora equivalente (LAeq), em dB (A), para os turnos da tarde (a) e noite (b) comparados a NBR 10.151/2000.

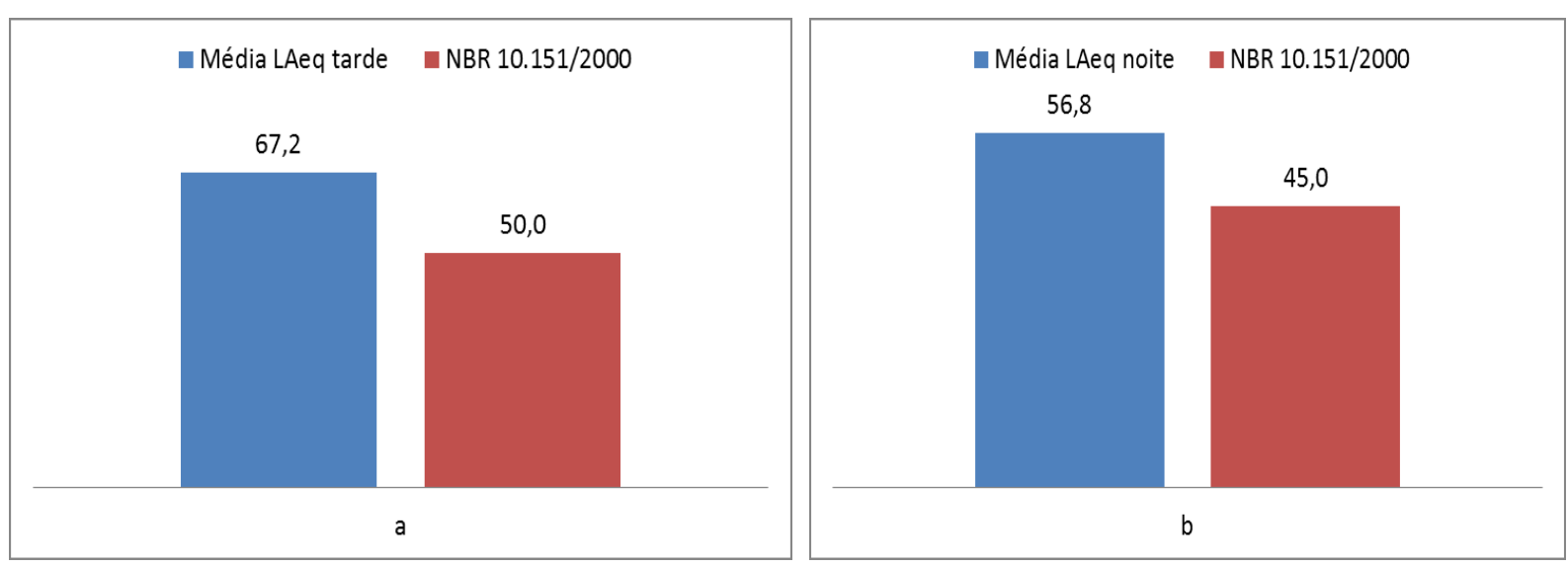

Comparando-se os dados acima com os valores de referência do Quadro 1 (NBR 10.151/2000), nas proximidades do hospital, percebe-se que o ruído encontrase acima do recomendado pela norma aqui exposta. A exemplo dos trabalhos de 
Penido, Azevedo e Souza (2011), Sousa et. al. (2010), Gonçalves e Adissi (2008), Nascimento et. al. (2007), Zannin e Szeremetta (2003), este trabalho revela, a inserção do município de Laranjal do Jari, nesta problemática que já vem sendo relatada pela literatura em que os níveis de pressão sonora equivalente assumem valores além daqueles estabelecidos pelos dispositivos legais vigentes.

\section{CONCLUSÕES}

Os valores do nível de pressão sonora equivalente, tanto à tarde quanto à noite, estão acima daqueles estabelecidos pela legislação nacional vigente.

Este dado torna-se relevante ao município, pois o executivo não dispõe de instrumento normativo que regule os níveis de ruído na cidade.

Face ao exposto, as pessoas que diariamente frequentam o local (na via ou hospital), ao fim do dia, podem apresentar estresse e cansaço físico e mental.

Neste sentido, a população do município deve conhecer tais problemáticas no sentido de garantir, para as mesmas, melhor qualidade vida.

\section{AGRADECIMENTOS}

À Pró-reitora de Pesquisa, Pós-graduação e Inovação do Instituto Federal do Amapá, pela bolsa de iniciação científica Jr. concedida ao aluno Jonatas Fernando Ramalho Cunha.

À Fundação de Amparo a Pesquisa do Amapá pela bolsa de iniciação científica Jr. concedida ao aluno Edivan Damascena Ribeiro.

\section{REFERÊNCIAS}

AMAPÁ (Prefeitura Municipal de Laranjal do Jari). Lei Municipal $\mathbf{n}^{\circ} \mathbf{2 6 1}$, de 22 de Julho de 2005. Institui o Código Ambiental do Município de Laranjal do Jari e dá outras providências.

ASSOCIAÇÃO BRASILEIRA DE NORMAS TÉCNICAS. NBR 10151: Acústica Avaliação do ruído em áreas habitadas, visando o conforto da comunidade Procedimento. Rio de Janeiro, 2000.

CARMO, L. I. C. Efeitos do ruído ambiental no organismo humano e suas manifestações auditivas. 1999. 45 p. Monografia (Especialização) - Centro de Especialização em Fonoaudiologia Clínica. Goiânia, 1999. 
COSTA, G. T. et. al. Descarte de óleos lubrificantes e suas embalagens nas oficinas mecânicas de Laranjal do Jari, Amapá. In: CONGRESSO NORTE NORDESTE DE PESQUISA E INOVAÇÃO (CONNEPI), 7., 2012, Palmas. Anais... Palmas: IFTO, 2012.

FREITAS, A. M. Estudo do impacto ambiental causado pelo aumento da poluição sonora em áreas próximas aos centros de lazer noturno na cidade de Santa Maria - RS. 2006. 97p. Dissertação (Mestrado em Engenharia Civil) Programa de Pós-Graduação em Engenharia Civil, Universidade Federal de Santa Maria. Santa Maria, 2006.

GIANINI, C. F. et. al. Avaliação do ruído ambiental: monitoramento e caracterização do ruído de fundo em Maringá - PR. Iniciação Científica CESUMAR. Maringá, v. 14 , n. 1, p. 55-62, jan-jun 2012

GONÇALVES, V. S. B.; ADISSI, P. J. Identificação dos níveis de pressão sonora em shopping center's na cidade João Pessoa. Revista Gestão Industrial. Ponta Grossa, v. 4, n. 3, p. 146-159, 2008.

HALLIDAY, D.; RESNICK, R.; WALKER, J. Fundamentos de Física 2. 4 ed. Rio de Janeiro: LTC, 1996, 284p.

MELO, R. A de. et. al. Previsão do nível de ruído gerado pelo tráfego de veículos na cidade João Pessoa, Brasil. In: CONGRESSO PAN-AMERICANO DE ENGENHARIA DE TRÁFEGO E TRANSPORTES E LOGÍSTICA (PANAM), 16., 2010, Lisboa.

Anais... Lisboa: IMT, 2010.

NASCIMENTO, R. G. et. al. Avaliação da poluição sonora na UNICAMP. Revista Ciências do ambiente On-Line. Campinas, v. 3, n. 1, 2007.

PENIDO, E. C.; AZEVEDO, F. R.; SOUZA, J. H. de. Poluição Sonora: aspectos ambientais e saúde pública. Viannasapiens. Juiz de Fora, v. 2, n. 1, p. 153-173, 2011.

QUADROS, F. S. Avaliação do ruído ambiental gerado por veículos de utilidade pública estudo de caso: caminhão de coleta de resíduos domiciliar. 144p.

Dissertação (Mestrado em Engenharia Elétrica) - Programa de Pós-Graduação em Engenharia Mecânica, Universidade Federal do Paraná. Curitiba, 2004.

SOUSA, L. H. C. D. et. al. Avaliação de ruído na região central de Maringá e Universidade Estadual de Maringá. Acta Scientiarum Technology. Maringá, v. 32, n. 1, p. 49-54, 2010.

ZANNIN, P. H. T.; SZREMETTA, B. Avaliação da poluição sonora no parque Jardim Botânico de Curitiba, Paraná, Brasil. Caderno de Saúde Pública. Rio de Janeiro, v. 19, n. 2, p. 683-686, 2003.

Artigo recebido em: 31/12/2014

Artigo aprovado em: 25/01/2016 